\title{
Extraction of Land Cover Units from Land Cover Components Based on Geometric Rules
}

\author{
Eliseo Clementini" ${ }^{\#}$, Stefano Natali ${ }^{*}$ \\ ${ }^{\#}$ Department of Industrial and Information Engineering and Economics, University of L'Aquila, Via G. Gronchi 18, I-67100 L'Aquila, Italy \\ E-mail:eliseo.clementini@univaq.it
}

"SISTEMA GmbH, Tiefer Graben 19 /2, A-1010 Vienna, Austria

E-mail:natali@sistema.at

\begin{abstract}
Land cover units are aggregations of land cover components that are obtained by using criteria of homogeneity and proximity of basic components. For example, residential urban settlements can be defined as aggregations of single buildings, neighboring green spaces, paved surfaces and small roads, which are separated by more prominent land cover components, such as main roads or rivers. Land cover components belong to standard classes typically obtained by an automated classification process applied to aerial or satellite images, such as buildings, constructed areas, bare soil, water, vegetation, and the like. Land cover units belong to more general classes, obtained by a combination of land cover components, such as residential areas, industrial areas, road networks, river systems, and agricultural units. In this paper, we describe an approach based on the application of geometric rules and semantic constraints to extract land cover units from land cover components. We use spatial operators to extract composite land cover units from land cover databases, where spatial operators are taken from standards of the Open Geospatial Consortium. Expert knowledge needs to be translated into specific automatic procedures, called complex object definitions or CODs. Finally, we build a prototype system, where the user can choose among a set of available CODs to build a sequence of actions that lead to the discovery of knowledge. We discuss several study cases, such as the recognition of urban settlements, agricultural land units, and road networks.
\end{abstract}

Keywords — land cover unit; land cover components; geometric rules; topological information.

\section{INTRODUCTION}

Extraction of land cover components (LCC), also called land cover objects, from satellite imagery or orthophotos is a critical task in many application domains. Roads, railroads, rivers, buildings, and lakes, extracted from "segmentation" and "classification" procedures, are typical examples of such features. Although these techniques yield important information from imagery, they do not yield to a higherlevel analysis, which allows for a true understanding of the role that individuals and groups of land cover components play in a process akin to human cognition. A land cover unit (LCU), also called land use object, is a high-level feature defined by the characteristic distribution of its constituent features and by its relationship with other land-use units and land cover components - its context. The context determines the rules for combining land cover objects into a community unit with a specific social function. Context is used as the basis for recognition.

In recent years a range of efforts has been made to extract high-level features from satellite imagery or orthophotos automatically. Most of the time the results are not reliable and have to be checked manually afterward. This manual feature extraction needs a lot of time to be performed correctly. For this reason, these tools are not widely used within the existing operational environment. On the other hand, digitizing is also time-consuming and, most of the times is a supplementary job for an imagery analyst (IA). If digitizing tasks could be done automatically, the IA would have more time for the real analysis and assembly of the final product. The operational world needs (semi) automatic feature extraction tools that are easy to use, and by changing only some parameters the tool should work in different areas.

The importance of semantic modeling in object discovery from satellite images has been remarked in the previous study [1]. A knowledge-base is used to assign segmented regions (i.e., extracted from images) into semantic objects (i.e., concepts of the knowledge-base) using a matching process between the regions and the ideas of the knowledgebase [2]. In recent years a range of efforts has been made to extract features from Earth Observation (EO) images automatically. Most of the time the results are not reliable or constant and have to be checked manually afterward. This manual feature extraction needs a lot of time to be performed correctly. The field called geospatial object-based image analysis (GEOBIA) has many common points with our 
approach [3]-[6]. In fact, GEOBIA's goal is the automated classification from land cover [7]-[11]: various techniques are used, mainly based on spectral analysis. If the latter is not sufficient to distinguish features, knowledge rules have been proposed [12]. Common GEOBIA software is Trimble eCognition (www.ecognition.com), Feature analyst (www.overwatch.com), and ENVI Feature Extraction Module (www.exelisvis.com). We believe that our approach is still different from GEOBIA because in GEOBIA spatial analysis GIS methods are seldom used.

Currently, private companies or public agencies, such as the Environmental Protection Agency of Austria in the Land Information System Austria (LISA) project [13]-[15], use mixing of automatic and manual techniques, ranging from domain expert consulting to image interpretation tools and to comparison with existing datasets. Overall, the techniques are time consuming and subjective. In other cases, semiautomatic systems have been applied like in the case of GMES Urban Atlas (www.eea.europa.eu/data-andmaps/data/urban-atlas), where the image analysis software eCognition has been used.

Image analysis software does not consider the structure of geometric components. In this paper, we propose the use of geometric operators, typically used in Geographical Information Systems (GIS), to check geometric properties that are independent of specific configurations. For example, topological relations can be used to inspect properties that are invariant concerning rotation, scaling, and continuous deformations. If we are interested in discovering buildings adjacent to a road, we might check for the topological relation 'touch' between a building and a road network.

The main idea pushed forward in this paper is the use of GIS spatial operators to extract composite land cover units from land cover databases. Spatial operators are taken from standards of the Open Geospatial Consortium (OGC) (www.opengeospatial.org). To reach the goal, it is essential to codify the expert knowledge to translate it into specific automatic procedures. The codification of expert knowledge is collected in complex object definitions (CODs). Each $\mathrm{COD}$ is a sequence of geometric operators that can recognize a specific geometric configuration.

We build a prototype system, called Topology Software System (TSS), where the user can choose among a set of available CODs to build a sequence of actions that can lead to the discovery of knowledge. We demonstrate the use of the application by discussing several study cases, such as the recognition of urban settlements, agricultural land units, and road networks. Preliminary results of this methodology were presented in [16]-[19].

In Section II, we preliminarily illustrate how LCUs can be built from geometric properties of LCCs; then, we introduce the methodology as a set of predefined functions and spatial operators and we describe the design and implementation of the STO software prototype. In Section III, we discuss the use cases that have been used to test the methodology and software implementation. Section IV gives short conclusions.

\section{MATERIALS AND METHODS}

\section{A. How LCUs are Built}

LCCs are the objects that belong to land cover: they represent the materials that cover a study site, such as vegetation, bare soil, rock, sand, and water. LCUs are the objects that belong to land use: they represent the way land is developed and used in terms of the types of activities allowed (agriculture, settlements, industries, and so on). LCUs are defined by the characteristic distribution of their constituent features, the LCCs (Fig. 1).
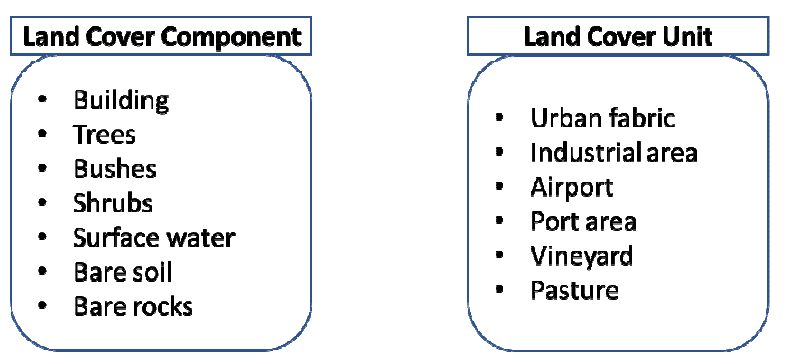

Fig. 1 Examples of LCCs and LCUs
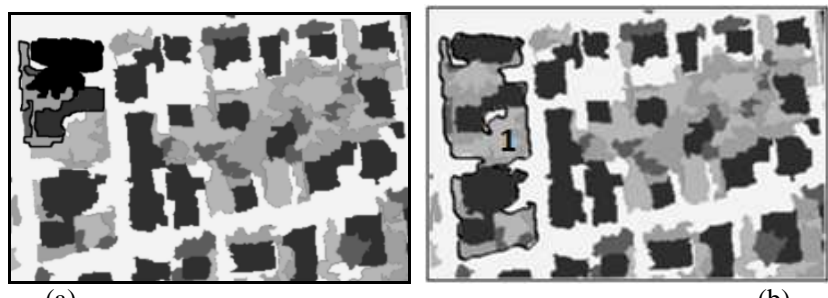

(a)

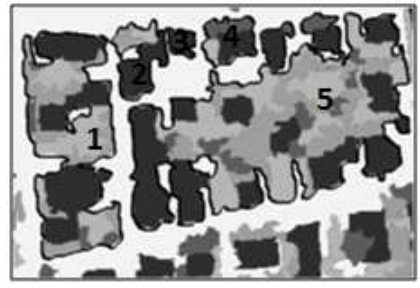

(c)

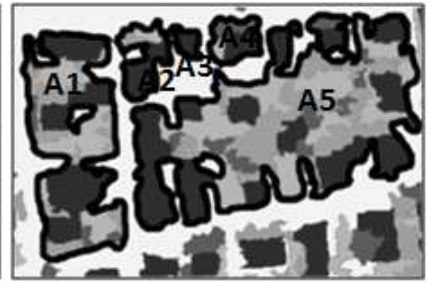

(d)

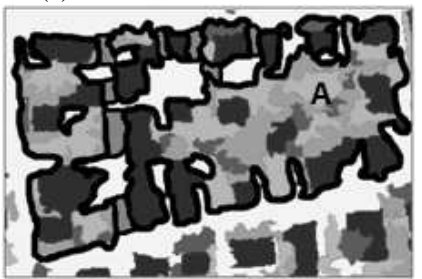

(e)

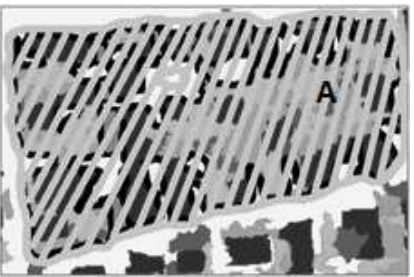

(f)

Fig. 2 Construction of a residential area LCU: (a) the aggregation process starts from the building in the upper left corner; (b) first aggregate is constructed; (c) all aggregates are constructed; (d) aggregates are grouped in one cluster; (e) fusion transforms the cluster in a single polygon; (f) shape regularization

It is critical to understand better the process of forming an LCU from LCCs. A residential area can be defined as an aggregate of buildings and neighboring parcels, such as gardens and parking lots, delimited by main roads or other LCUs of different types. The LCU residential area is built using roads as basic delimiting factor: only the "important" roads are used for this. Smaller roads, which are only used as access roads to buildings, are not used as the boundary of the LCU geometry but are inside it. 


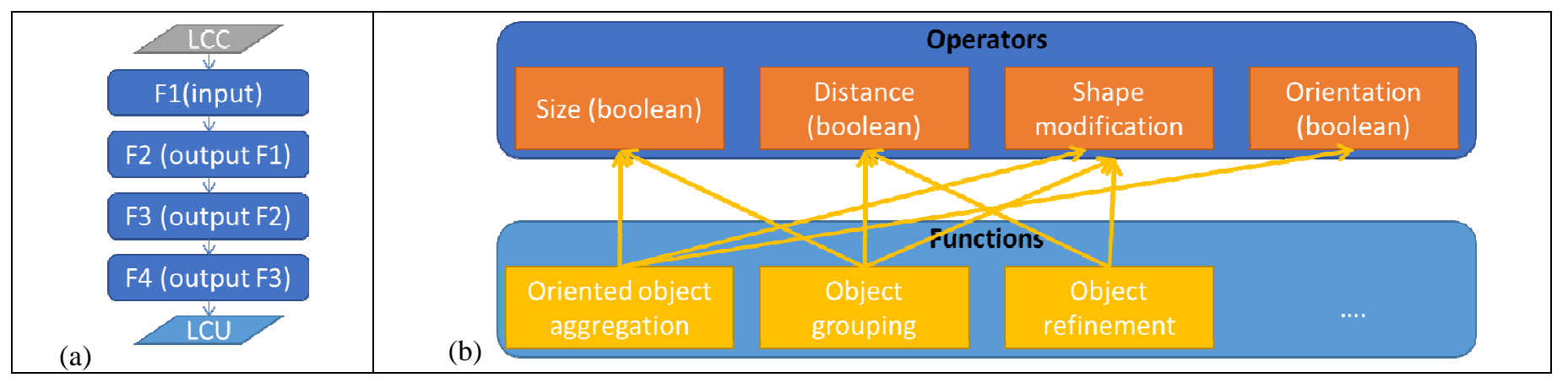

Fig. 3 The methodology to build LCU: (a) a sequence of functions; (b) each function makes use of spatial operators

TABLE I

DEVEloped SPATIAL Operators

\begin{tabular}{|l|l|l|}
\hline Operator & Type & Description \\
\hline Is small/medium/ big (size) & Boolean & Check the size of objects with respect to a reference size \\
\hline Merge & Shape modification & $\begin{array}{l}\text { To create a single object from a set of objects in touch (internal } \\
\text { borders removal) }\end{array}$ \\
\hline Is elongated (shape) & Boolean & checks whether an object has an elongated shape \\
\hline Same shape orientation & Boolean & Check if two elongated objects have the same orientation \\
\hline Is close/ medium/ far (distance) & Boolean & $\begin{array}{l}\text { Check the distance between two objects with respect to a reference } \\
\text { distance }\end{array}$ \\
\hline Fusion & Shape modification & Join into a single object different objects within a given distance \\
\hline Smoothing & Shape modification & Modify the shape of an object removing small imperfections \\
\hline
\end{tabular}

Buildings are judged based on their size: only small/medium buildings can represent residential usage, while larger ones might represent industrial or commercial areas. The residential area also includes other neighboring LCCs, such as green areas and groups of trees. A further aspect to distinguish residential areas could be the proximity to paved surfaces non-belonging to a road network, such as parking lots.

Therefore, in our methodology, we evaluate LCUs residential areas with a spatial analysis based on geometric operators. We analyze the shape and size of buildings and the spatial relations between them. The first process to carry out is to aggregate LCCs that semantically belong to specific land cover classes (e.g., "building," "tree," "bushes," and "herbaceous"), which are dimensionally comparable and not too big or small with respect to a reference size and recursively satisfy the topological relation "touch". The touch relation is satisfied when two objects' geometries have at least one boundary point in common but no interior points. The process starts considering a single LCC that belongs to the "building" class and that is small/medium in size concerning given reference size. It continues by aggregating the chosen building LCC with other LCCs that belong to classes like "tree," "bushes" and "herbaceous" (i.e., gardens) that are comparable in size and that are in "touch" relation (Fig. 2a). The aggregation process continues by adding other LCCs that satisfy previous constraints with the constructed object so far until the residential area is surrounded by the LCC category "other constructed area" (Fig. 2b). The previous steps are repeated starting from another "building" object that is not included in any urban settlement aggregate already constructed (Fig. 2c). The aggregation process so far used the "size" operator to compute the land cover objects' size, the "touches" operator to check the topological relation "touch", and the "merge" operator to aggregate land cover objects' geometries that are in "touch". Each aggregate found in this phase is an object geometry of class Polygon (see OGC categorization in which elements of the class Polygon are simple polygons possibly having holes).

After previous aggregates are found, they need to be grouped together to identify residential areas. Clustering algorithms, based on boundary or centroid distance, can be used to identify the aggregates that can be grouped in a single cluster. The resulting geometry is, in this case, belongs to the class Multi Polygon in the OGC categorization (a multi polygon is made up of disconnected components, each of them being a polygon) (Fig. 2d). We use a "distance" operator to select aggregates that are at close distance and the "union" operator to group objects" geometries into a single geometry collection.

The next step is to obtain a single geometry for the LCU residential area, by making a fusion of the previously obtained grouped object and by making the convex hull of neighboring parts of boundaries (Fig. 2e). Also, regularization of the object shape can be performed, by filtering small irregularities (filling small concavities) and by replacing irregular sides with straight edges (Fig. 2f). Finally, for the obtained object, we can test if its size is larger than a Minimal Measuring Unit (MMU) for the LCU residential area and discard the smaller objects. For example, on our data set, the MMU for residential areas was set to $1000 \mathrm{~m}^{2}$.

\section{B. Methodology}

Each class of LCU needs a specific definition coming from expert knowledge. Each LCU is defined by a COD, which is a sequence of calls to predefined functions (such as 'aggregate,' 'group,' 'refine'). In turn, each function makes calls to spatial operators as needed (Fig. 3). 
By changing the order of functions and the parameters passed on to them, it is possible to define new CODs. Predefined functions are typical transformations of spatial data, as suggested in Section 2, by examining the residential area LCU. Accurately, to implement the residential area use case, the sequence of functions that must be carried out is the following: (1) Object Aggregation; (2) Object Grouping; (3) Object Refinement; (4) Object Size Check.

The function "Object aggregation" is used to recursively aggregate LCCs on a binary spatial relation. In the case of the residential area, the function worked on the topological relation "touch" to form an aggregate of LCCs for which there is a connected path of "touch" relations. Further, the function can specify the size of components that can take part in the aggregate, e.g., they should be dimensionally comparable and not too big or small with respect to a reference size and they belong to specific LCC classes. The function "Object grouping" is used to group together objects that satisfy some distance criteria. Clustering algorithms, based on boundary or centroid distance, may be used. This function uses geometric operators to check the distance among simple objects and the "union" operator to group a set of geometries in a single output geometry. The function "Object Refinement" is used to merge the components (e.g., by adding corridors where the boundaries are closer), which constitute the result of calls to the Object grouping function that are not connected. Also, the refine function is used to remove small parts or irregularities, such as concavities or holes, obtaining a smooth shape. The function "Object size check" can be used at the end of other steps to exclude results that do not satisfy the MMU.

Other functions can be designed to satisfy the needs of another use case. Spatial operators that are invoked by functions can be distinguished in those already implemented in OGC standards, such as topological relations, Euclidean distance, set operations, convex hull, and many others, and those for which it does not exist a standard implementation: regarding this latter group, we implemented our own version of operators (some of them are listed in Table 1). For more information on spatial operators, it is possible to refer to the broad literature on them, such as topological relations [20, 21], projective relations [22]-[26], directional relations [27], and visibility relations [28]-[31].

\section{The TSS Software Prototype}

The TSS software prototype has been implemented as a web-GIS architecture. Fig. 4 describes the use case model for TSS, where three main use cases were identified in the analysis phase: "Manage Earth Observation Model," the function for management of the ingested data model, "Define Complex Object," the function for defining new LCUs, and "Identify Complex Object," the function for identifying LCUs on the ingested data model that satisfy the COD. Fig. 5 shows the main scenario for the "Identify Complex Object" use case in a sequence diagram.

A logical model is a static view of the objects and class that make up the design/analysis space. Fig. 6 shows the domain model (a component of the logical model) for TSS. Every data model ingested in the TSS is coded as an "Earth Observation Model" that aggregates a set of TSSObject which represent the "feature" concept defined in the Geography Mark-Up Language (GML). The LCU and LCC concepts were modeled using the TSSComplexObject and TSSSimpleObject respectively: we used a common design pattern defined "composite". A composite is a group of objects in which some objects may contain other objects (LCUs) so that some objects represent groups and others represent individual items or leaves (LCCs). TSSComplexObject shares with other complex objects a common definition that is modeled as an ordered aggregation of functions.

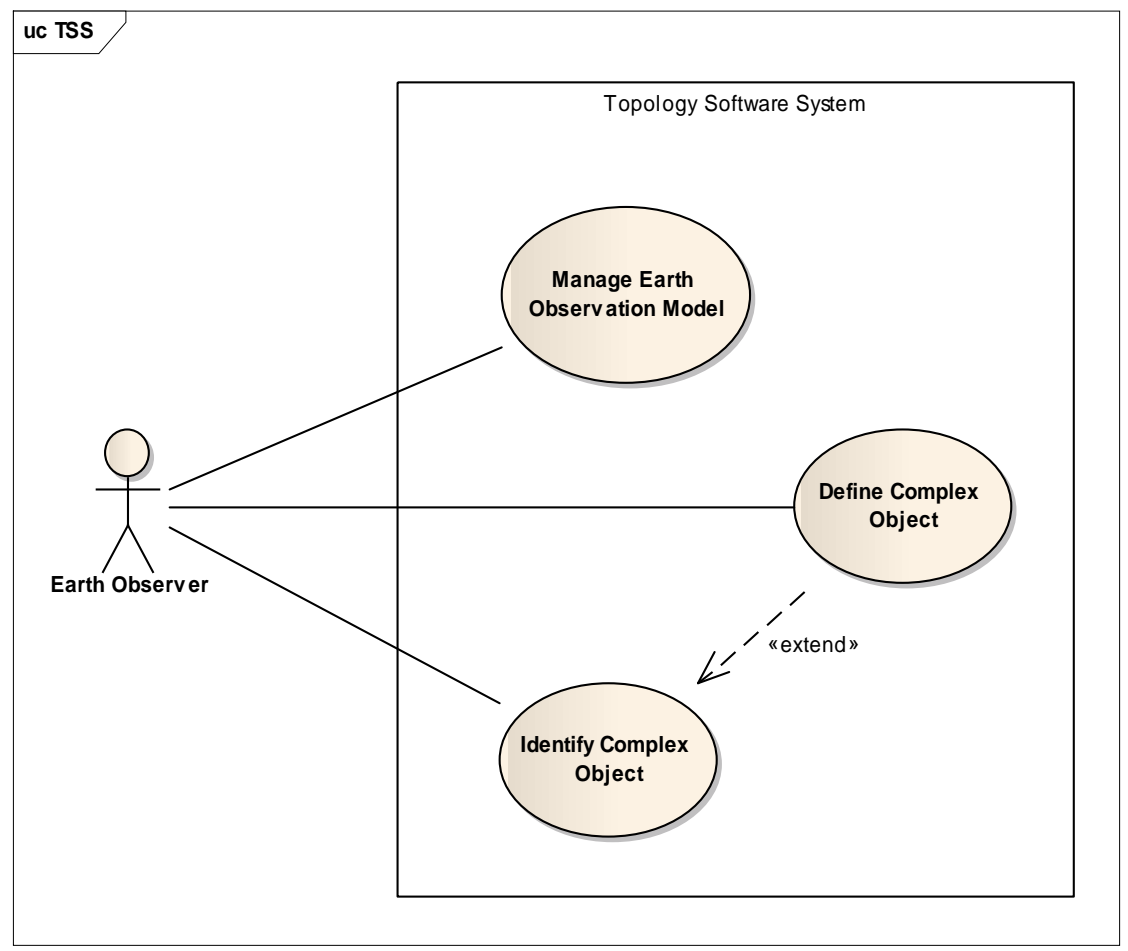

Fig. 4 The use case model for TSS 


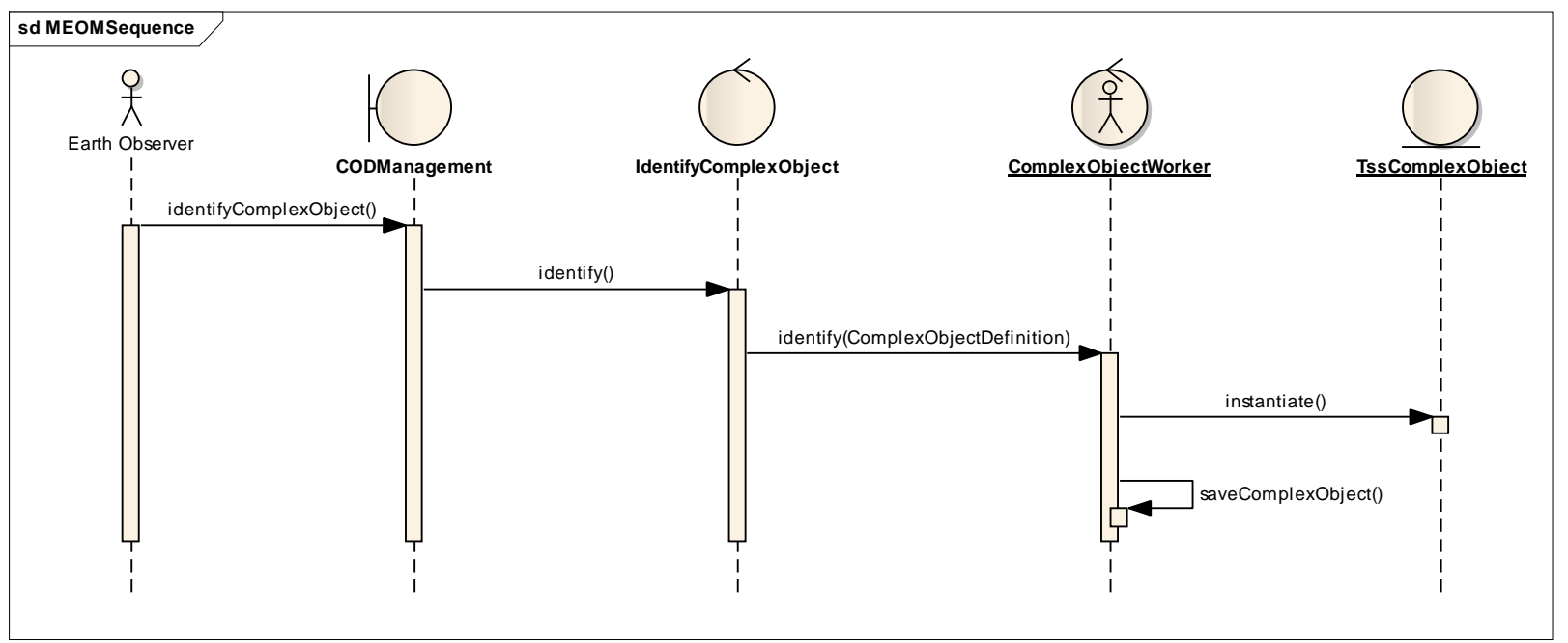

Fig. 5 The sequence diagram of the "Identify Complex Object" use case

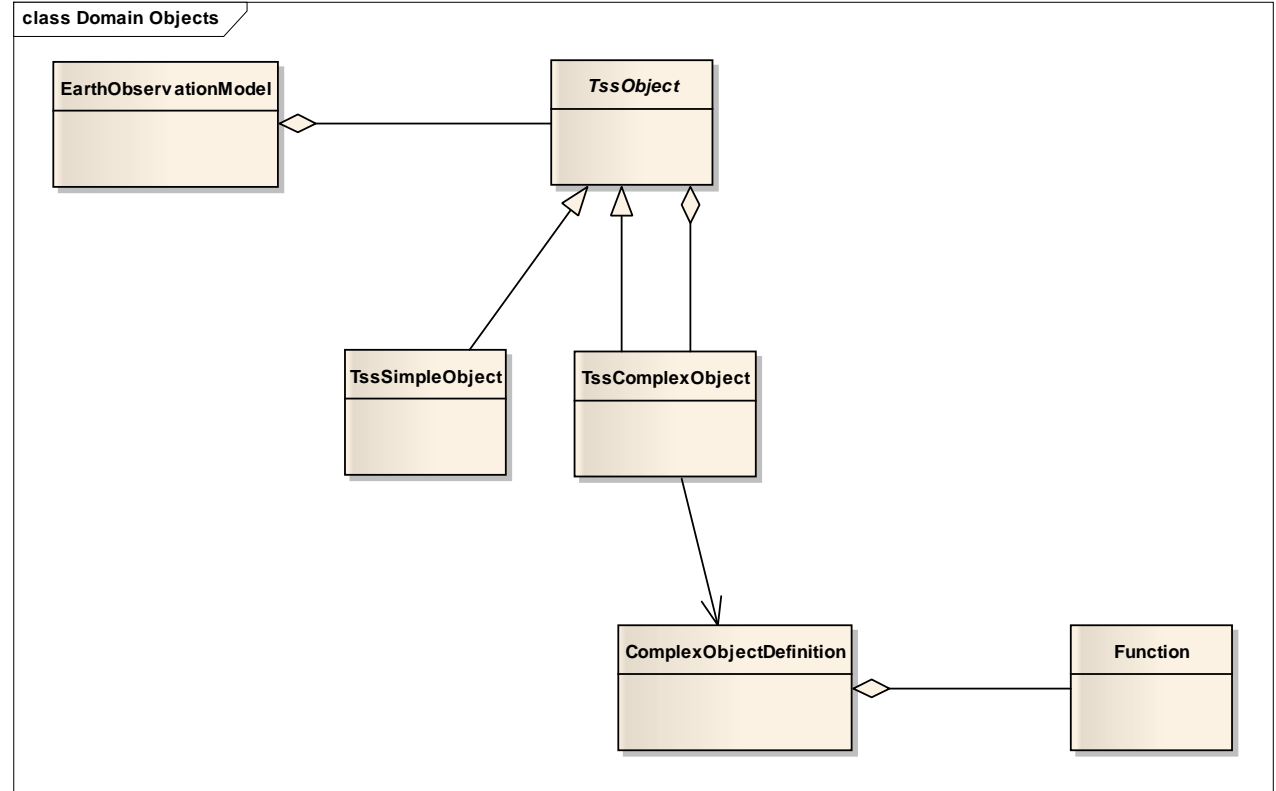

Fig. 6 The domain model of TSS

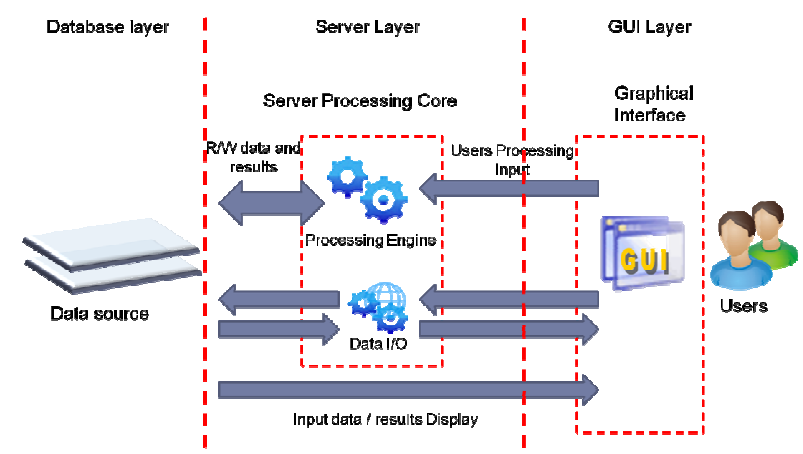

Fig. 7 The three-layered TSS architecture

The TSS architecture, structured in three layers, is summarized in Figure 7. The GUI layer is the direct interface toward end-users. The GUI allows users to load an LCC dataset into the system for processing and visualizing it (Fig. 8); then, it allows the COD definitions and the application of function sequences to identify LCUs (Fig. 9).
The server layer is the core of TSS: it contains all modules for $\mathrm{I} / \mathrm{O}$ processes and all modules for the application of CODs to the ingested data model. The topological operator module provides spatial operators and functions organized like a library. The database layer contains three main databases: the data archive, which hosts the input datasets and the identified LCUs, the CODs database, which stores the already developed definitions, and the tiles database, which contains all the tiles for each input dataset as well as those for the output layers. We decided to use a standard web map tiling service to make the input and output data visualization fast and multi-resolution.

\section{RESULTS AND DISCUSSION}

To test the developed prototype, we chose our use cases among two data projects, the Land Information System of Austria (LISA) and the European Urban Atlas project. The objective of LISA is to achieve a consensus on a new Austrian land information system and demonstrate its benefits offering improved spatial and thematic content. 
LISA is designed to serve common land monitoring needs providing information on the status quo and the changes occurring in Austria's landscape. The Urban Atlas project is part of the local component of the GMES/Copernicus land monitoring services. It provides reliable, inter-comparable, high-resolution land use maps for 305 Large Urban Zones and their surroundings for the reference year 2006. It was created to fill a gap in the knowledge about land use in European cities.

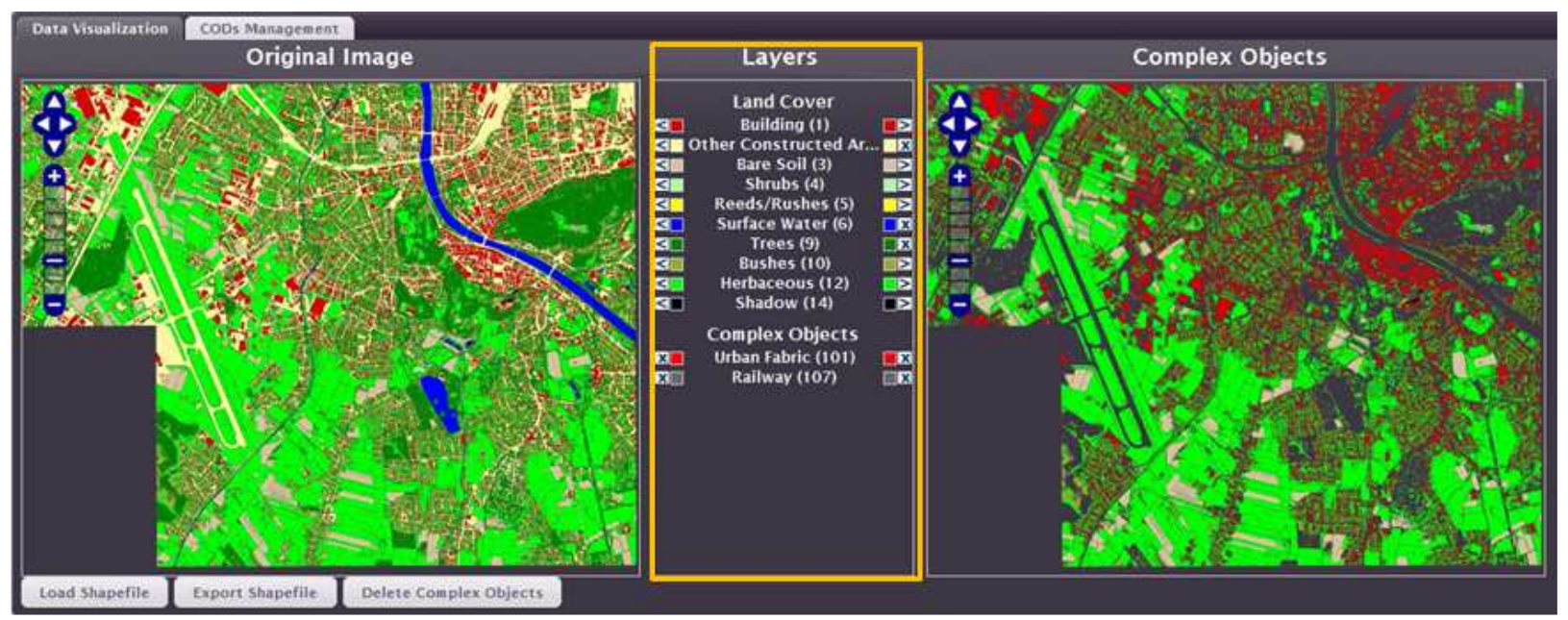

Fig. 8 The GUI displays both the original dataset and the output LCUs

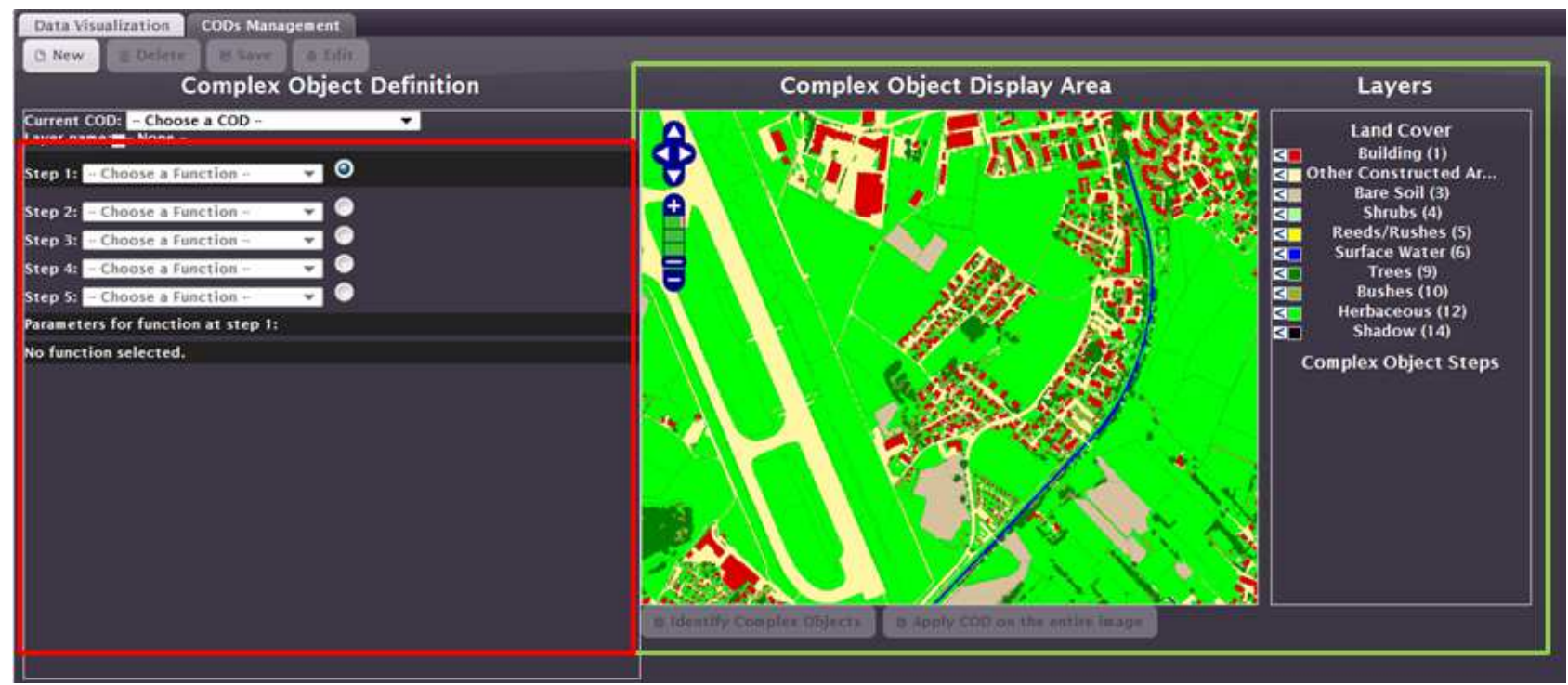

Fig. 9 The GUI for the new COD definition

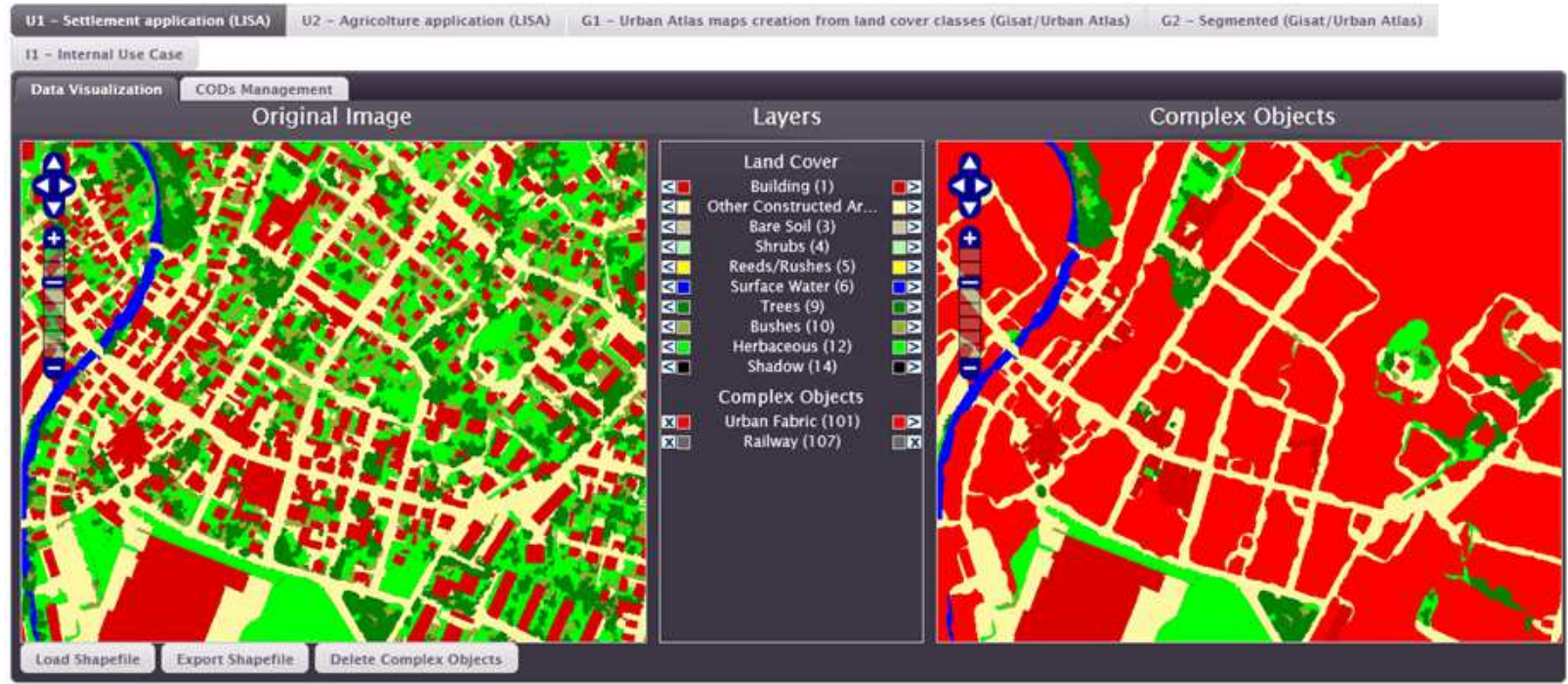

Fig. 10 The use case of residential areas 


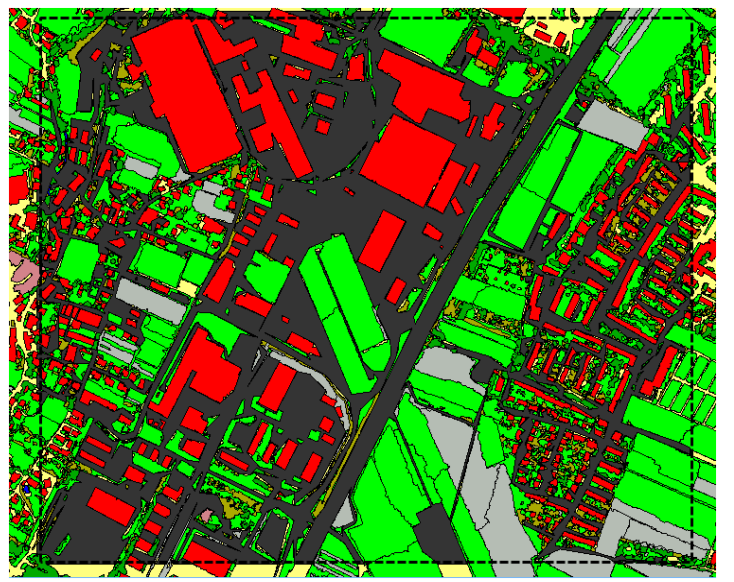

(a)

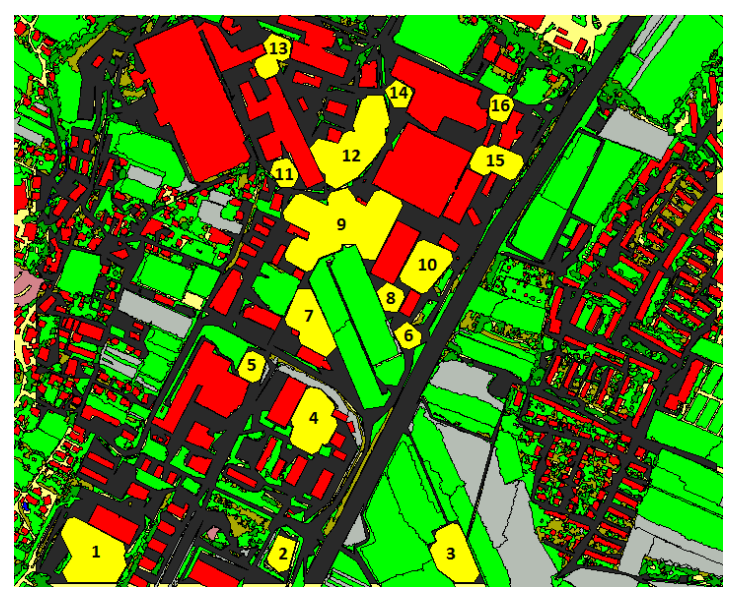

(b)

Fig. 11 Identification of parking lots from LCCs (a) to LCUs (b)

As a first use case, we already discussed the procedure to find the COD for residential areas. In Fig. 10, we can see the result of running the COD on a fraction of Salzburg on the LISA dataset. A similar use case is the identification of industrial or commercial areas. From human expert knowledge, we understood that these areas are characterized by having large parking spaces around. Therefore, one step in their identification consists in finding out large parking lots. We defined the COD for finding parking lots as the paved LCCs that are larger than a road and that have a compact or elongated shape. In this COD, a buffer operation has been applied twice, first as an inward buffer to find areas larger than the road and then as an outward buffer of the same width to identify the parking lots. The results are shown in Fig. 11.

A different use case was chosen in agriculture. Similarly, to urban areas, the candidate agricultural objects are, in a first step, differentiated using roads, forest borders and rivers as basic delimiting factor. An additional constraint was introduced after talking to experts that suggested considering the orientation of the field as well since the same orientation implies homogeneous cultivation. Therefore, we built a COD that was able to group together fields with approximately the same orientation. The agricultural use case finds single cultivation LCUs by aggregating LCCs belonging to the same class of cultivation and neighboring each other. The sequence of functions that have been identified in this use case is the following: (1) Object Aggregation; (2) Non-Oriented Object Inclusion; (3) Object
Check Size. The Object Aggregation function finds out agricultural aggregates, joining LCCs that semantically belong to specific crop classes and satisfy the topological relation "touch"; further, they have a similar (and well defined) orientation (Fig. 12a). In addition to the Boolean touch operator and merge operator, this function uses geometric operators to compute the shape orientation of LCCs. The "Non-Oriented Object Inclusion" function serves to aggregate the LCCs with agricultural field label with nonclear orientation (e.g., objects of square or circle shape) with the agricultural aggregates identified using the previous function with which they have the highest number of "touch" relations and with which they have the same crop class (Fig. 12b). The last consideration is useful to preserve a compact form of agricultural aggregates. Finally, the Object Check Size function carries out a validation of the land use objects constructed so far. Only agricultural aggregates that have an area greater than the MMU of the output data set are considered valid. In this case the value of MMU for valid agricultural land use objects is established at $5000 \mathrm{~m}^{2}$.

A last test case that we illustrate in this paper is the construction of a river system LCU. A river system is made up of the river and parts of the river banks including LCCs of various types (bare soil, rocks, bushes, and so on) if the average width of the river system is maintained. The COD to build a river system invokes the aggregation and grouping functions. Also, a splitting function is needed to split parts of larger objects neighboring the river. In the aggregate function, all water LCCs that are elongated in shape were analyzed to check if they are part of the same river and to filter other small water component. Other small LCCs that are in touch are aggregated with the water system. In the group function, all the components obtained that belong to the same river are joined to obtain a single river system LCU. The result is shown in Fig. 13.

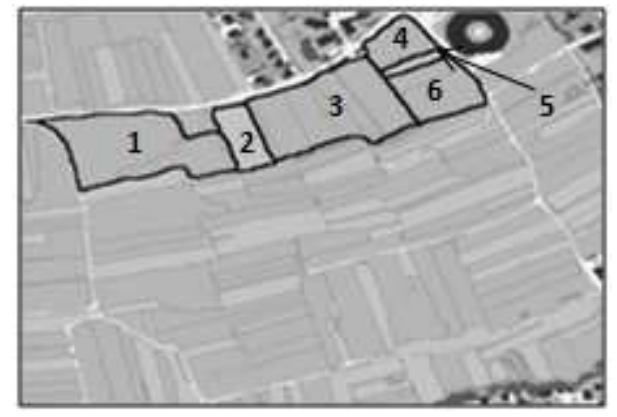

(a)

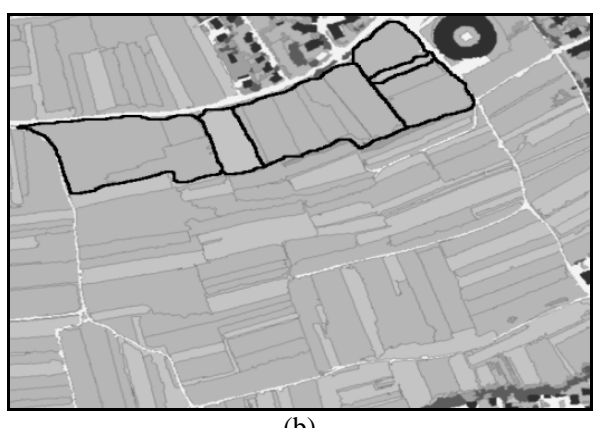

(b)

Fig. 12 Identification of agricultural areas: (a) agricultural aggregates; (b) refinement applying the Non-Oriented Object Inclusion function 


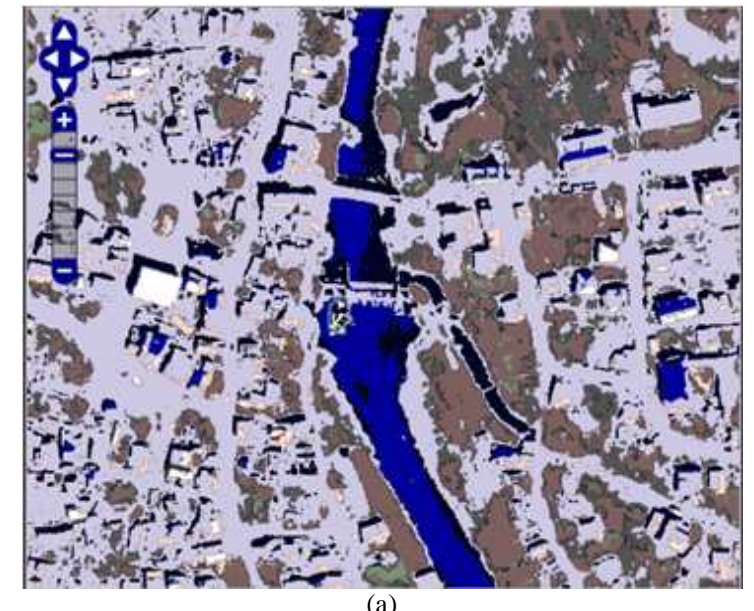

(a)

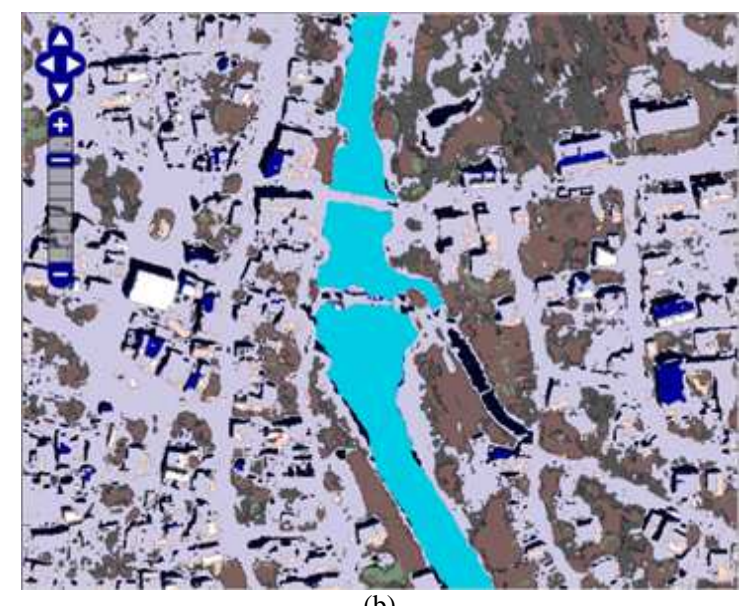

(b)

Fig. 13 Identification of a river system: (a) the original dataset; (b) the resulting $\mathrm{LCU}$

\section{CONCLUSION}

There is a lot of interest in the process of extracting meaningful objects from land cover data sets. LCCs contains information on the material that is found on a given component, but the semantics of complex objects can only be added by expert knowledge in each context. Though, a lot of information is intrinsically contained in the geometry of LCCs and their spatial relations: analyzing the geometric information can help to identify LCUs. In this paper, we proposed a methodology to define the recurrent geometric properties of LCUs and reach their construction via a sequence of predefined functions and spatial operators. Some of the spatial operators are available in OGC standard complying systems and other spatial operators needed a custom development. We developed a prototype web-GIS application that facilitated the testing of the methodology. An initial evaluation has been performed by comparing the results of the use cases to internal expert knowledge. As reported in [16-19], LCUs could be recognized with an average overall accuracy of more than $85 \%$. Further work is needed to make wider user tests to compare the results of our methodology with other existing techniques.

\section{ACKNOWLEDGMENT}

The authors would like to thank the European Space Agency (ESA) for granting the Support to Topology (STO) project

(http://rssportal.esa.int/deepenandlearn/tikiindex.php? page=STO+Project), the SISTEMA $\mathrm{GmbH}$ (http://www. sistema.at/), Vienna, Republic of Austria, for developing the prototype of the Topology Software System (TSS), the Environmental Protection Agency of Austria (http://www.umweltbundesamt.at/en/), Department for Biodiversity and Nature Conservation, Vienna, Republic of Austria, for providing LISA images and test cases, the GISAT (http://www.gisat.cz/content/en), Prague, Czech Republic, for providing Urban Atlas images and test cases.

\section{REFERENCES}

[1] D. Guo, H. Xiong, V. Atluri, and N. R. Adam, "Object Discovery in High-Resolution Remote Sensing Images: A Semantic Perspective," Knowledge and Information Systems vol. 19, pp. 211-233, 2009.

[2] G. Forestier, A. Puissant, C. Wemmert, and P. Gançarski, "Knowledge-based region labeling for remote sensing image interpretation," Computers, Environment and Urban Systems, vol. 36, pp. 470-480, 2012.

[3] H. Thunig, N. Wolf, S. Naumann, A. Siegmund, and C. Jürgens, "Automated LULC classification of VHR optical satellite data in the context of urban planning," in Geographic Object-Based Image Analysis GEOBIA 2010. vol. XXXVIII-4/C7, E. A. Addink and F. M. B. Van Coillie, Eds., ed Ghent, Belgium: ISPRS, 2010, p. 6.

[4] E. S. Malinverni, A. N. Tassetti, and A. Bernardini, "Automatic land use/land cover classification system with rules based both on objects attributes and landscape indicators," in Geographic Object-Based Image Analysis GEOBIA 2010. vol. XXXVIII-4/C7, E. A. Addink and F. M. B. Van Coillie, Eds., ed Ghent, Belgium: ISPRS, 2010, p. 6.

[5] T. Novack, H. J. H. Kux, R. Q. Feitosa, and G. A. Costa, "Per block urban land use interpretation using optical VHR data and the knowledge-based system Interimage," in Geographic Object-Based Image Analysis GEOBIA 2010. vol. XXXVIII-4/C7, E. A. Addink and F. M. B. Van Coillie, Eds., ed Ghent, Belgium: ISPRS, 2010, p. 6.

[6] G. Chen, Q. Weng, G. J. Hay, and Y. He, "Geographic object-based image analysis (GEOBIA): emerging trends and future opportunities," GIScience \& Remote Sensing, vol. 55, pp. 159-182, 2018.

[7] E. P. Baltsavias, "Object extraction and revision by image analysis using existing geodata and knowledge: current status and steps towards operational systems," ISPRS Journal of Photogrammetry \& Remote Sensing, vol. 58, pp. 129-151, 2004.

[8] Y. Liu, Q. Guo, and M. Kelly, "A framework of region-based spatial relations for non-overlapping features and its application in objectbased image analysis," ISPRS Journal of Photogrammetry \& Remote Sensing, vol. 63, pp. 461-475, 2008.

[9] M. J. Barnsley, L. Møller-Jensen, and S. L. Barr, "Inferring Urban Land Use by Spatial and Structural Pattern Recognition," in Remote Sensing and Urban Analysis, J.-P. Donnay, M. J. Barnsley, and P. A.Longley, Eds., ed: Taylor \& Francis, 2001, pp. 102-130.

[10] M. Hussain, C. Davies, and R. Barr, "Classifying Buildings Automatically: A Methodology," presented at the GISRUK 2007: Proceedings of the Geographical Information Science Research UK 15th Annual Conference, Maynooth, Ireland, 11th-13th April 2007, 2007.

[11] J. Wijnant and T. Steenberghen, "Per-Parcel Classification of Ikonos Imagery," presented at the $7^{\text {th }}$ AGILE conference on Geographic Information Science, Heraklion, Greece, 2004.

[12] Z. Rongqun and Z. Daolin, "Study of land cover classification based on knowledge rules using high-resolution remote sensing images," Expert Systems with Applications, vol. 38, pp. 3647-3652, 2011.

[13] R. Grillmayer, G. Banko, J. Scholz, C. Perger, K. Steinnocher, A. Walli, et al., "Land Information System Austria (LISA) Objektorientiertes Datenmodell zur Abbildung der Landbeckung und Landnutzung," in Angewandte Geoinformatik 2010 - Beiträge zum 22. AGIT-Symposium, J. Strobl, T. Blaschke, and G. Griesebner, Eds., ed Salzburg, Austria: Wichmann, 2010, pp. 616-621.

[14] R. Prüller, R. Grillmayer, G. Banko, R. Mansberger, K. Steinnocher, W. Stemberger, et al., "Nutzen von innovativen Technologien für eine flächendeckende, flexible Landbeobachtung Österreichs," in Angewandte Geoinformatik 2011 - Beiträge zum 23. AGIT- 
Symposium, J. Strobl, T. Blaschke, and G. Griesebner, Eds., ed Salzburg, Austria: Wichmann, 2011, pp. 239-244.

[15] J. Weichselbaum, G. Banko, C. Hoffmann, M. Riedl, M. Schardt, K. Steinnocher, et al., "Land Information System Austria (LISA) Bedarfsgerechte Landnutzungsinformationen für die öffentliche Verwaltung," in Angewandte Geoinformatik 2009: Beiträge zum 21. AGIT-Symposium, J. Strobl, T. Blaschke, and G. Griesebner, Eds., ed Salzburg, Austria: Wichmann, 2009, pp. 492-497.

[16] E. Ippoliti, E. Clementini, S. Natali, and G. Banko, "A methodology for the automatic generation of land use maps," in GI Forum 2012. Geovisualization, Society and Learning, Salzburg, Austria, July 3 - 6, ed Berlin: Wichmann Verlag, 2012, pp. 456-465.

[17] E. Ippoliti, E. Clementini, and S. Natali, "Automatic generation of land use maps from land cover maps " in Proceedings of the AGILE'2012 International Conference on Geographic Information Science, Avignon, April 24-27, 2012.

[18] S. Natali, E. Clementini, E. Ippoliti, G. Banko, and L. Brodsky, "Topology software system: Support to the creation of land use maps," presented at the ESA-EUSC-JRC 2012. Image Information Mining Conference: Knowledge Discovery from Earth Observation Data, German Aerospace Center (DLR), Oberpfaffenhofen, Germany, 24-26 October, 2012.

[19] E. Clementini and E. Ippoliti, "Automatic extraction of complex objects from land cover maps," in Geographic Information Science at the Heart of Europe - Proceedings of the 16th AGILE International Conference on Geographic Information Science. vol. Lecture Notes in Geoinformation and Cartography, D. Vandenbroucke, B. Bucher, and J. Crompvoets, Eds., ed Berlin: Springer, 2013, pp. 75-93.

[20] E. Clementini and A. G. Cohn, "RCC*-9 and CBM*," in Geographic Information Science. 8th International Conference, GIScience 2014, Vienna, Austria, September 24-26, 2014. Proceedings. vol. LNCS 8728, M. Duckham, E. Pebesma, K. Stewart, and A. U. Frank, Eds., ed Berlin: Springer, 2014, pp. 349-365.

[21] F. Tarquini and E. Clementini, "Spatial relations between classes as integrity constraints," Transactions in GIS, vol. 12, pp. 45-57, 2008.

[22] R. Billen and E. Clementini, "Semantics of collinearity among regions," in On the Move to Meaningful Internet Systems 2005: OTM Workshops - 1st Int. Workshop on Semantic-based Geographical Information Systems (SeBGIS'05), Agia Napa, Cyprus, October 31 -
November 4, 2005. vol. 3762, R. Meersman, Z. Tari, and P. Herrero, Eds., ed Berlin: Springer-Verlag, 2005, pp. 1066-1076.

[23] R. Billen and E. Clementini, "Projective relations in a 3D environment," in Geographic Information Science: 4th International Conference, GIScience 2006, Münster, Germany, September 20-23, 2006. vol. 4197, M. Raubal, H. Miller, A. Frank, and M. Goodchild, Eds., ed Berlin: Springer, 2006, pp. 18-32.

[24] E. Clementini, "Projective Relations on the Sphere," in Advances in Conceptual Modeling - Challenges and Opportunities - ER 2008 Workshops - 2nd International Workshop on Semantic and Conceptual Issues in GIS (SeCoGIS 2008). vol. 5232, I.-Y. Song, Ed., ed Berlin Heidelberg: Springer-Verlag, 2008, pp. 313-322.

[25] E. Clementini, S. Skiadopoulos, R. Billen, and F. Tarquini, "A reasoning system of ternary projective relations," IEEE Transactions on Knowledge and Data Engineering, vol. 22, pp. 161-178, 2010.

[26] E. Clementini and R. Billen, "Modeling and computing ternary projective relations between regions," IEEE Transactions on Knowledge and Data Engineering, vol. 18, pp. 799-814, 2006.

[27] E. Clementini, "Directional relations and frames of reference," GeoInformatica, vol. 17, pp. 235-255, 2013.

[28] P. Bartie, E. Clementini, and F. Reitsma, "A Qualitative Model for Describing the Arrangement of Visible Cityscape Objects from an Egocentric Viewpoint," Computers, Environment and Urban Systems, vol. 38, pp. 21-34, 2013.

[29] P. Fogliaroni and E. Clementini, "Modeling Visibility in 3D Space: a Qualitative Frame of Reference," in $3 D$ Geoinformation Science: The Selected Papers of the 3D GeoInfo 2014. vol. LNG\&C, M Breunig, M. Al-Doori, E. Butwilowski, P. V. Kuper, J. Benner, and K. H. Haefele, Eds., ed Berlin: Springer, 2015, pp. 243-258.

[30] P. Fogliaroni, J. O. Wallgrün, E. Clementini, F. Tarquini, and D. Wolter, "A Qualitative Approach to Localization and Navigation Based on Visibility Information," in Spatial Information Theory, 9th International Conference, COSIT 2009, Aber Wrac'h, France, September 2009. vol. 5756, K. Stewart Hornsby, C. Claramunt, M. Denis, and G. Ligozat, Eds., ed Berlin: Springer, 2009, pp. 312-329.

[31] F. Tarquini, G. De Felice, P. Fogliaroni, and E. Clementini, "A Qualitative Model for Visibility Relations," in 30th Annual German Conference on Artificial Intelligence (KI 2007). vol. 4667, J. Hertzberg, M. Beetz, and R. Englert, Eds., ed: Springer, 2007, pp. 510-513. 